\title{
Prevalence of Stunting and Its Associated Factors Among Children 6-59 Months of Age in Pastoralist Community, Northeast Ethiopia: A Community Based Cross Sectional Study
}

\section{Awrajaw Dessie ( $\square$ awrajawdss@gmail.com )}

University of Gondar College of Medicine and Health Sciences https://orcid.org/0000-0001-5436-9936 Mulugeta Gebreayohanes

University of Gondar College of Medicine and Health Sciences

\section{Research}

Keywords: Stunting, Children, Pastorals, Afar region, Ethiopia

Posted Date: July 28th, 2020

DOI: https://doi.org/10.21203/rs.3.rs-48559/v1

License: (a) (1) This work is licensed under a Creative Commons Attribution 4.0 International License. Read Full License 


\section{Abstract}

Background: Globally, stunting affects over 161 million children under the age of five, and one million deaths each year. Stunting hits hard on African and Asian children. In Ethiopia, the level of stunting is critically high and remains a major public health challenge among those under the age of five. Numerous factors such as poor maternal health and nutrition, inadequate feeding practices for infants and young children, deficiencies in micronutrients, infections and environmental health conditions are related to stunting. There is, however, dearth of evidence in Ethiopia's pastoral populations.

Methods: A community-based cross-sectional study was conducted from 2-31 January 2018 to assess the prevalence and associated factors of stunting among children 6-59 months of age in Dubti District, Afar Region, north East Ethiopia. For this study, a total of 554 children were included, and multi-stage sampling technique was used to select the study participants. Data was entered in EPI-Info version 7.2 and height for age was converted to Z-score with WHO Anthro software. A binary logistic regression analysis was conducted to identify factors associated with stunting. The significance of the associations was determined at $\mathrm{p}$-value $<0.05$ and the adjusted odds ratio at $95 \% \mathrm{Cl}$ was calculated to evaluate the strength of the associations.

Results: The prevalence of stunting was 39.5\% (95\% Cl: 35.4-43.5\%). The odds of stunting was increased, so does age of the child increased as compared to 6-11 months of children. Initiating breast feeding after 1 hour after birth (AOR $=1.99 ; 95 \% \mathrm{Cl}: 1.22,3.23)$, not exclusively breast feeding for at least 6 months $(\mathrm{AOR}=2.57 ; 95 \% \mathrm{Cl}: 1.49,4.42)$, poor dietary diversity $(\mathrm{AOR}=1.93 ; 95 \% \mathrm{Cl}: 1.03,3.62)$, and using unprotected water sources for drinking $(A O R=1.68 ; 95 \% \mathrm{Cl}: 1.21,2.94)$ were significant factors.

Conclusion: Stunting levels in pastorals was critically high among children aged 6-59 months. The study showed that stunting was associated with different nutritional and non-nutritional factors. Intersectoral coordination is required to tackle stunting by enhancing the community's safe water supply, optimal breast feeding practice, food diversity and economic status.

\section{Background}

Malnutrition is one of the most important causes of infant morbidity and mortality in developing nations. Sub-Saharan Africa is contributing to high rates of malnutrition-related infant morbidity and death [1]. It influences the potential economic competitiveness of a nation, and remains a crucial concern in developing countries [2]. Stunting is a chronic malnutrition that occurs when children fail to achieve their linear growth potential and cause severe irreversible physical and cognitive damage upon them [3]. It will have also irreparable effects on the future development of a child, will increase the vulnerability of populations and weaken their ability to cope with food stress episodes\{Jember, 2014 \#1;Jember, 2014 \#1;Jember, 2014 \#1;Jember, 2014 \#1;Jember, 2014 \#1;Mihret, 2014 \#1;International., 2012 \#4\} [4].

Globally, stunting affects more than 161 million children under the age of five, with an estimated one million related child deaths per annum. Stunting hits African and Asian children hard [3]. Under-nutrition 
in Ethiopia is one of the main health problems for children under five years of age [5]. Stunting costs in Ethiopia $16.5 \%$ of GDP per annum [6]. Stunting can have catastrophic effects that last a lifetime [7].

Ethiopia is one of the poorest countries in the world, with very weak human development indices. Around 23 million Ethiopians live significantly below the minimal poverty line under conditions and food insecurity remains a major problem [8]. The Afar region's production system is dominated by pastoralists (90\%), from whom agro-pastoralists (10\%) now emerge after some permanent and temporary rivers on which small-scale irrigation develops. The recurrent episodes of drought and unseasoned flooding and disease outbreaks in Ethiopia's pastoral areas are strong reminiscences of how poverty reduction, food security and pastoral livelihood strategies remain largely dependent on the climate system and susceptible to seasonal

The prevalence of stunting in Ethiopia has dropped considerably from 58\% in 2000 to $38 \%$ in 2016, but in the Afar region the stunting level is above the national average of 41 percent [9]. Malnutrition in the Afar region poses a huge problem [10]. While the consequences of stunting are clear, its causes are more complex [11]. Direct factors contributing to stunted growth and development include poor maternal health and nutrition, inadequate infant and young child feeding practices, micronutrient deficiencies, and infections [12].

The sustainable development goals (SDGs) identified stunting, along with other nutrition indicators, as a target to reduce global malnutrition; meeting this goal demands renewed and concerted efforts focused on disparity [13]. Stunting is regarded by the Ethiopian government as a major public health issue and an obstacle to its economic goals. The Health Sector Transformation Plan (HSTP), part of GTP II, aimed at reducing mortality rates of 30 per 1,000 live births below five years in Ethiopia; reducing stunting to $26 \%$ in less than 5 years [14].

Since stunting is highly dependent on local geo-cultural factors such as tradition and community livelihoods, it is important to consider its prevalence and causative factors in this context to prioritize development interventions to mitigate the problem. There is however a lack of evidence in the pastoralist communities. These communities give priority to access to seasonal pastures and they are highly mobile between various wet and dry seasons to seek food for their livestock - their main livelihoods, in a very scattered manner. Therefore, this study in the district of Dubti may involve planning long-term decisionmaking and treatment measures for the results of stunting prevalence and its association factors; rather, targeting short-term food assistance that would not solve stunting in the study area. This study will therefore provide input on prevalence and associated factors for children aged 6-59 months to local level government officials, NGOs, policy makers working to reduce the child mortality rate contributing to the goal of HSTP and GTP II of the Ethiopian country.

\section{Methods And Materials}

\section{Study Design and Period}


A community based cross-sectional study was conducted from January 2-31, 2018.

\section{Study Area}

The study was conducted in Dubti district, Afar region, Northeast Ethiopia (Figure 1). It is one of the 32 districts in Afar region. It is located approximately $7 \mathrm{~km}$ far from regional capital Semera [15] and 600 km northeast of Addis Ababa, capital of Ethiopia [16]. The study area is constantly struck by drought. The community largely they are pastorals who have a small plot of land for cultivation and are engaged largely in the rearing or husbandry of livestock. They are living in a dispersed manner and many areas remain remote and difficult to access. The pastorals are highly vulnerable to widespread droughts, loss of seasonal and perennial river and groundwater access, migratory, and have very limited access to basic healthcare and nutritional services [15]. According to the district health centre annual report, a total of 8187 under five children were residing in the area [17].

\section{Source and Study Population}

Source populations were all children aged 6-59 months living in the district and those children from 4 (3 rural and 1 urban) randomly selected kebels were the study population. Children who have been seriously ill during the entire data collection season and children with spinal curvature (kiphosis, scoliosis and kiphoscoliosis) have been excluded.

\section{Sample Size Determination and Sampling Technique}

The sample size was determined by single population proportion formula using the assumptions of $95 \%$ confidence level; proportion of stunting among 6-59 months children in Afambo district 32.2\% [18]; 5\% margin of error; design effect of 1.5 . The final sample size was therefore calculated to be 555 , after a $10 \%$ non-response rate was added. A multi-stage sampling technique was used to select the study participants. In the first stage, from the two urban kebeles, one kebele was selected by the lottery method, and in the same way, from the 12 rural kebeles, three kebeles were selected. Second, the total sample size was allocated proportionally on the basis of the total number of households with children aged 6-59 months and a simple random sampling technique was used to select children on the basis of the existing sampling frame from health posts. The mother or guardian has been interviewed.

\section{Variables measurement}

Height and weight: The height of infants aged between 6 months and 23 months was measured in recumbent position to the nearest $0.1 \mathrm{~cm}$ using a board with an upright wooden base and a movable headpiece. Children between 24 and 59 months of age were measured in a standing position of $0.1 \mathrm{~cm}$ to the nearest. In addition, the child weight was measured using an electronic digital weight scale for 
children who were comfortable to measure on their own, and also for children who were uncomfortable to measure on their own, we used the combined mother and child weight and the mother's individual weight to calculate the child's weight [20].

Stunting: Height-for-age is a measure of linear growth retardation and cumulative growth deficits. Children whose height-for-age Z (HAZ) score is below minus two standard deviations (-2 SD) from the median of the reference population were considered to be stunted. Children below minus three standard deviations (-3 SD) considered to have been severely stunted [9].

Economic status of household: Since the community is pastoral, the economic status of households has been measured using the Tropical Livestock Unit (TLU) as a proxy. TLU is a measure developed by the Food and Agriculture Organization (FAO) that allows the combination of multiple animal species into a weighted measure of total body weight and potentially market value [21]. A single animal weighing 250 $\mathrm{kg}$ is a single TLU, which provides a weighting factor of 0.7 for cattle, 0.1 for sheep, 0.1 for goats and 0.01 for chickens. The economic status of households was determined by comparing the TLU scores to the standard ranking. A score below 5 TLU shows the household is poor. A TLU score of 5 to 12.99 showed the household's economic status was medium and richer households ranked 13 and above TLU [21].

Minimum Dietary Diversity Score (MDDS): Proxy for the adequacy of dietary micronutrient density for infants and young children. Consumption of 4 or more of the 7 food groups means that the child is likely to consume at least one animal food source and at least one fruit or vegetable in addition to the staple food (grains, roots, or tubers) in the last 24 hours. Four food groups should be drawn from the list of seven food groups: grains, roots and tubers; legumes and nuts; dairy products (milk yogurt, cheese); meat , fish , poultry and liver / organic meat; eggs; vitamin A-rich fruits and vegetables; and other fruits and vegetables [18].

\section{Data Quality Management}

The structured questionnaire was prepared in English and translated into Afar language and translated back into English by language experts to check its consistency. Pre-test of the questionnaire was performed on $5 \%$ of the sample size in the similar area-Asayita district, Afar region. The weight measurement scale was checked against zero readings after and before each child was weighed. A two day training was given to data collectors and supervisors on procedures, techniques, and ways of collecting data. Besides, a clear introduction was provided about the purpose and objectives of the study to the respondents before data collection. In addition, continuous and strict supervision and on the spot checking was carried out during the process.

\section{Data processing and analysis}


The data was checked, coded, and entered in to Epi-Info version 7.2. software. Data on sex, age, height, and weight was transferred with participants' identification number to WHO Anthro software to convert nutritional data into $Z$ scores of the indices HAZ using the WHO standard. Afterwards, the data, including the HAZ was exported for analysis to SPSS version 20 software. The bivariate analysis was performed to determine the association of stunting and associated factors and the variables were selected for multivariate analysis by $p$-value $<0.2$. A multivariable binary logistic regression analysis was employed to control the possible effects of confounders. The model goodness of fit test was checked by Hosmer and Lemshow Test, and it was found fit $(\mathbb{2} 2=11.57, \mathrm{p}$-value $=0.17)$. Finally, variables which showed significant associations were identified on the basis of the adjusted odds ratios (AOR) with a $95 \% \mathrm{Cl}$ and $\mathrm{p}$-value $<0.05$.

\section{Results}

\section{Demographic and Socio-Economic Characteristics}

The study included a total of 554 study participants, giving a response rate of 99.82 percent. The majority were male headed by 510 (92.1 percent) households. Approximately 513 (92.6\%) respondents were Muslim, and more than three-fourth (79.1\%) of the participants were Afar by ethnicity. Five hundred and seven mothers (91.5\%) were married and 437 (79.2\%) aged between 20-34 years. Most of them (57.9\%) can't read and write. Four hundred twelve (74.4\%) of households have been classified as poor based on TLU. Average household size was 5 ( $S D \pm 1.81$ ), where $45.3 \%$ of households had fewer than 5 family members. Nearly half (48\%) had one child under five and $46.4 \%$ of households had two children under five years of age. Most of the mothers were housewives, 347 (62.5\%), and $452(81.6 \%)$ of the fathers were occupational agro-pastoralists. The majority of households in 514 (92.8\%) were not productive safety net programme (PSNP) users (Table 1).

\section{Child Characteristics and Child Carrying Practice}

This study showed that $312(56.5 \%)$ of the children were males and $111(20.0 \%)$ of them were aged 6-11 months with 31(IQR 19) months of median age. More than one third of children (35.7 percent) were second by birth order and $549(99.1 \%)$ of the children were singletons. More than half $(52.5 \%)$ of the children were born at home, according to this study. The study revealed that $439(79.2 \%)$ mothers started breastfeeding with colostrum within one hour immediately after a birth and $80.5 \%$ of the majority of children were breastfed exclusively for at least six months. This study found that 303 (54.7\%) children had a meal frequency of at least 3 meal frequencies a day, and that the majority of 461 (83.2\%) had a minimum dietary diversity score of $<4$. About the $56.7 \%$ of children have been fully vaccinated (Table 2 ).

\section{Maternal Characteristics and Health Service Utilization}


This study shows that more than half (50.4\%) of mothers were aged between 26 and 35 years when they gave birth to the index child, with an average age of 28 years (IQR 6). Only half of mothers $(50.2 \%)$ completed the full ANC schedule and three-fourth (75.1\%) received PNC. One hundred and eighty nine $(34.1 \%)$ mothers during their pregnancy or lactation had no extra meal at all. Husbands make decisions in the majority of households concerning the use of money (71.2\%) (Table 3 ).

\section{Environmental Health Characteristics of Households}

The majority of households in 345 (62.3\%) used public tap as a source of drinking water, which is one of the improved sources of drinking water. Two hundred and sixty seven (48.2\%) households had access to water for the round trip within less than 15 minutes. Three hundred twenty seven participants $(59.2 \%)$ used latrine. From the study, 214 mothers and caregivers (38.6\%) washed their hands with water only (Table 4).

\section{Prevalence of Stunting}

Stunting was found to have a prevalence of $39.5 \%$ (95\% Cl: $35.4-43.5 \%)$. The prevalence was $29.6 \%$ and $9.9 \%$, respectively of moderate and severe stunting. The prevalence of stunting among female and male children was $36.0 \%$, and $42.3 \%$. The highest prevalence of stunting was $61.1 \%$ among children aged 36 59 months, $41.2 \%$ among those aged $24-35$ months, $34.4 \%$ among those aged $12-23$ months, and the lowest $14.4 \%$ was among children aged $6-11$ months. Of stunted children, the majority $41.8 \%$ were between the ages of 36-59 months and the minimum 5.5\% were between the ages of 6-11 months.

\section{Factors associated with stunting}

Stunting was correlated with wealth status, infant age, breast feeding initiation, complementary feeding, minimum dietary diversity ranking, and source of drinking water. Children from poor households were 5.5 times more likely to be stunted than rich family children ( $A O R=5.50 ; 95$ per cent $\mathrm{Cl}: 2.52,12.04)$. Stunting was more common among children aged 12-23, 24-35, and 36-39 months compared to children aged 611 months $(\mathrm{AOR}=2.55 ; 95 \% \mathrm{Cl}: 1.27,5.09),(\mathrm{AOR}=3.02 ; 95 \% \mathrm{Cl}: 1.58,5.78)$, and $(\mathrm{AOR}=4.12 ; 95 \% \mathrm{Cl}$ : $2.00,8.45)$, respectively.

Breast feeding initiation and exclusive breast feeding have been found to be predictors for stunting in the study area. Chances of being stunted have increased by $99 \%$ among children who started breast feeding after 1 hour compared with children who started breast feeding within 1 hour of birth (AOR $=1.99 ; 95 \% \mathrm{Cl}$ : $1.22,3.23)$. In comparison, infants who only had breast feeding for less than 6 months were 2.57 more likely to be stunted than their counterparts who had had breast feeding for 6 months or longer (AOR = $2.57 ; 95 \% \mathrm{Cl}: 1.49,4.42)$.

Minimum dietary diversity score (MDDS) has also been found to be linked to stunting. Children from mothers who had 4 MDDS and less were $93 \%$ more likely to be stunted than their counterparts who had 
more than 4 MDDS (AOR $=1.93 ; 95 \% \mathrm{Cl}: 1.03,3.62)$. Using unprotected river water in children's households was $68 \%$ more likely to be stunted than households receiving drinking water from public tabs $(\mathrm{AOR}=1.68 ; 95 \% \mathrm{Cl}: 1.21,2.94)($ Table 5$)$.

\section{Discussion}

The children's stunting level for 6-59 months in the district of Dubti, Afar, was 39.5\%. The prevalence of severe and mild stunting was $9.93 \%$ and $29.6 \%$, respectively. The level of stunting is designated to be very serious or critical in the study area, according to the WHO classification [22], which implied that stunting in Ethiopia remains a major public health challenge. The result is in line with the national prevalence of stunting, which shows $38 \%$ prevalence of stunting among under five children [23]. However, the prevalence was lower than other studies conducted in the district of Hadibu Abote, Oromia region, reported to be 47.6\% [24]; in the district of Bule Hora, South Ethiopia, 47.6\% [19]; and 67.8\% in the district of Asayita district, Eastern Ethiopia [25]; 56.6\% in the district of Medebay Zana district, Northern Ethiopia [26]; 49.1\% in the district of Libo-Kemekem, North-west Ethiopia [27]. However, the finding was higher than the prevalence of stunting which was reported in Afambo district of Eastern Ethiopia, 32.2\% [18]; the study done in Dollo Ado district 34.4\% [28], another study conducted in eastern Ethiopia which showed prevalence of $34.4 \%$ [29, 30]; a study conducted in Delanta district in Ethiopia which reported a $22.1 \%$ prevalence of stunting [31]. The discrepancy could be due to differences in the participants' geographic characteristics, period of study and other socio-economic characteristics. The consumption of milk and milk products in the study area could help to children growing and thereby minimize stunting, as verified by the scholarly article [32]. This could be helpful to fight for improving nutritional status of children in the nomadic community, where a large population of cattle, sheep, goats, and camels are found.

Our study shows, as the child's age increases, so is the likelihood that it will stunt. Scholarly articles in Ethiopia and elsewhere in other parts of the globe [33-35] support the finding. Because stunting has a persistent and cyclical aspect, poor nutritional practice, weaning, lower and insufficient breast and complementary feeding methods have been undermined and become ineffective as the age of the child rises, this further induces stunting. Another possible explanation for increased risk of stunting in older children may be due to unhygienic preparation of supplementary foods which exposes children to recurrent infections. Restricted access to safe drinking water in the study area often exposes these children to varied types of infections and diarrheal diseases that raise the risk of chronic malnutrition even further.

In addition, this study indicated that household children who were poor in economic status were at higher risk of stunting. The finding is supported by similar studies in Ethiopia [34, 36], and elsewhere in Africa [35]. It is well understood that poor people are suffering from poor diet, inadequate schooling, poor clothing, poor hygiene and health, resulting in children suffering from failures in growth [34]. Policies seeking to increase the number of animals in the nomadic culture are therefore crucial in combating malnutrition. Increasing animal health by raising awareness about the prevention and control of animal 
diseases, improving access to animal health facilities and, most importantly, piling up animal fodder is necessary.

This study suggested that children born from households who drink from unimproved water sources (river) were at higher risk of stunting compared to children from households who drink from safer sources. The finding is corroborated by similar studies in Ethiopia, which showed that households who drink water from an unprotected source were more stunted than their counterparts [26, 34]. The possible explanation could be lack of safe water that exposes these children to varied types of infections and diarrheal diseases that increase the risk of chronic malnutrition even further. Improving access to improved water sources is very important to combat the problem of malnutrition in the area. The study stressed that non-nutrition-specific interventions are of paramount importance in improving the problem.

In this study it is found that early initiation of breast feeding for the inborn is associated with stunting. Children whose mothers started breastfeeding within one hour after birth were less suffering from stunting. It is generally recommended that children start breast feeding immediately after birth to improve the child nutritional status. This may be because early breastfeeding initiation helps for effective breastfeeding via increased secretion and breast milk output which will provide the developing baby with adequate nutrient like colostrum [31]. Colostrum provides the infant with natural immunity and also has an input in reducing hypoglycaemia and hypothermia which in turn has a devastating effect on the infant's health status $[31,37]$. This study re-affirms the WHO recommendation which underscores the significance of timely breastfeeding on child health [32]. This finding is supported by similar studies conducted in Tigray, Northern Ethiopia [26]; in Indonesia [38]. These findings show early initiation of breastfeeding as a method of early-life maternal treatment and best nutrient that can minimize the risk of stunting. Early breastfeeding is one of the entrances to successful later breastfeeding and ensures that children are provided enough food intake [39]. Therefore, in order to promote child health and improve the nutritional status of children, health education should be provided to mothers about the value of breastfeeding their infants immediately after birth, during post-natal care, and by health extension workers and the women health development armies.

Moreover, the study revealed that exclusive breast feeding is one of the significant predictors of stunting. Children who hadn't been breastfed exclusively for at least 6 months were 2.57 times more likely to get stunted. The study corporates similar studies performed in different areas among under five children [4043]. The probable reason is that inappropriate timing for introducing certain kinds of complementary food to a child can affect its nutritional status because its digestive and immune systems are not yet mature. Previous implementation of supplements, in particular under unhygienic conditions, may be a major cause of malnutrition [43]. Exclusive breastfeeding is very important, especially in the area where the sanitation status is very low to prevent infections that could impede the child's development. For its benefit, therefore, the mothers should be advised and the enabling environment should be established which promotes optimal breastfeeding. 
This study suggested that lack of sufficient food diversity was found to be a stunting indicator. Children who have consumed less optimal dietary diversity are $93 \%$ more likely to be stunted. Various similar studies conducted in Ethiopia and elsewhere support this finding [44-46]. Therefore this study indicates that malnutrition can be minimized by increasing complementary food dietary diversity. Households should be trained and encouraged to include sufficient and diverse foods that can meet the child's need for energy and nutrients. Since pastoral community owns a large number of cattle, it is imperative to provide their children with milk.

The study has the limitations set out below. Because of the studies cross sectional design, we cannot declare a temporal relationship between the stunting and other independent variables. Standard procedures have been used for height / length measurement but errors in measurement are inevitable, especially within assessors. In addition, a recall bias can occur in the reporting age of Children live in rural villages. Nevertheless, we have tried to confirm the age reported in the immunization card if available.

\section{Conclusion}

In conclusion, the stunting condition had been critically high in the pastoralist community that indicates stunting is still the public health concern. The study revealed that nutrition specific and non-nutrition specific factors were related to stunting. Age of child, economic status of household, breast feeding initiation period after birth, exclusive breast feeding and water supply were found to be the key predictors of stunting. It is recommended to boost household economic status by maintaining animal welfare and diversification of income sources, by encouraging optimum practice of feeding infants and young children by complying with $\mathrm{WHO}$ and national guidelines on breast feeding and complementary feeding. In fact, it is also important to protect the existing water sources from possible contaminations and increasing the coverage of safe water sources in the area. Thus, intersectoral collaboration is of paramount importance for addressing the various dimensions of stunting correlates. Given the multifaceted nature of the causes of stunting in this study, there is need to address all causes of stunting in an integrated manner as well.

\section{Abbreviations}

ANC: Antenatal Care; AOR: Adjusted Odds Ratio; Cl: Confidence Interval ; COR: Crude Odds Ratio; GDP: Gross Domestic Product; GTP: Growth Transformation Plan; HAZ: Height for Age Z-score; HSTP: Health Sector Transformation Plan; IQR: Inter Quartile Range; MDDS: Minimum Dietary Diversity Score; NGO: Non-Governmental Organization; PNC: Postnatal Care; SD: Standard Deviation; SDG: Sustainable Development Goal; SPSS: Statistical Package for Social Science; TLU: Tropical Livestock Unit; WHO: World Health Organization

\section{Declarations}

Acknowledgments 
The authors would like to extend their gratitude acknowledgments to the University of Gondar, College of Medicine and Health Sciences, Institute of Public Health for providing ethical clearance. We also would like to appreciate the Afar Regional Health Bureau for logistic support and facilitating the data collection process. Finally, we would like to extend our cordial

appreciation to all the participants and data collectors.

\section{Competing interests}

The authors declare that they have no competing interests.

\section{Authors' contribution}

$A D$ : Involved in the data analysis, interpreted results and discussions and wrote up of the draft manuscript and review the manuscript draft and the corresponding author. MG: Initiated the research concept, wrote up of the research proposal and involved in the data analysis and presentations of results. Both authors have read and approved the final manuscript.

\section{Funding}

No funding received for this study, except the logistic support for data collection process from Afar Regional Health Bureau.

\section{Availability of data and materials}

The necessary data are included in the paper datasets and the full blond of the data set can be available with reasonable request from the correspondent author on reasonable request.

\section{Ethics approval and consent to participate}

Ethical clearance was obtained from University of Gondar, Institute of Public Health, Institutional Review Board. The letter of support was also sought from the Afar Regional Health Bureau, the Dubti District Health Office, and the kebele administration units. Verbal consent was obtained from the parents / caretaker of study subjects and they were explained the study objective. The privacy and confidentiality of the information obtained was safeguarded at all levels. Where case of any sick child or child with severe nutrition during field work has been observed, advice has been given to the parents / care taker so that, in addition to nutritional consultation, they can take to the nearest health institution.

\section{Consent for publication}

Not applicable.

\section{References}


1. Olack, B. and e. al, Nutritional Status of Under-five Children Living in an Informal Urban Settlement in Nairobi, Kenya. 2011.

2. CSA and a. ICF, 2011 Ethiopian Demographic and Health Survey Findings. 2012: Calverton, Maryland, USA: CSA and ICF International.

3. Mercedes de Onis, F.B., Childhood stunting: a global perspective. Matern and Child Nutrition 2016(PMID: 27187907; PubMed Central PMCID: PMC5084763).

4. Mihret J, T.A., Disaster Risk Management and Sustainable Development Department. Bahirdar University. Bahirdar, Ethiopia. 2014.

5. Yisak, H., T. Gobena, and F. Mesfin, Prevalence and risk factors for under nutrition among children under five at Haramaya district, Eastern Ethiopia. 2015.

6. Burney, T., M. Martinez, and K. Nnamdi, Achieving Food and Nutrition Security in Ethiopia. Findings from the CARE Learning Tour to Ethiopia. 2014: 1825 Street, NW, Suite 301.Washington, DC. p. 3-15.

7. WHO, Levels and Trends in Child malnutrition. UNICEF / WHO / World Bank Group Joint Child Malnutrition Estimates Key findings of the 2016 edition; Available at URL:http://www.who.int/nutgrowthdb/estimates. 2016.

8. Group, A.D.B., Federal Democratic Republic of Ethiopia Country paper 2016-2020. 2015.

9. CSA and a. ICF, Ethiopia Demographic and Health Survey 2016. 2017.

10. EDHS, Ethiopia Mini Demographic and Health Survey. 2014: Addis Ababa, Ethiopia. p. 52-57.

11. IFPRI, Global nutrition report 2016: From promise to impact: Ending malnutrition by 2030. 2016, International Food Policy Research Institute Washington, DC.

12. Organization, W.H., Global nutrition targets 2025: policy brief series (WHO/NMH/NHD/14.2). Geneva: World Health Organization, 2014.

13. UN-DESA, The Sustainable Development Goals Report 2017. 2017.

14. MoH, Health Sector Transformation Plan (HSTP) 2015/16 - 2019/20. 2015: Addis Ababa, Ethiopia. p. 3-40.

15. Sarson, M., ECHO Support to Community Management of Malnutrition Vital for Children's Health in Ethiopia. 2013: p. 1-2.

16. Amodu, E.E.U.a.O.K., Complementary Feeding Practices among mothers and Nutritional Status of Infants in Akpabuyo Area Cross River State Nigeria. 2016.

17. Center, D.W.H., Dubti Woreda Health Center 2016 Annual Report. 2016.

18. Legesse, M., T. Abuhay, and Y. Haile, Determinants of Child Malnutrition among Agro Pastorals in Northeastern Ethiopia: A cross-sectional Study. Southern east Ethiopia. Research Article. iMedPub Journals., 2016. 10(4:15): p. 4-7.

19. Asfaw, M., et al., Prevalence of undernutrition and associated factors among children aged between six to fifty nine months in Bule Hora district, South Ethiopia. BMC Public Health, 2015: p. 1-41.

20. Organization, W.H., Physical status: The use of and interpretation of anthropometry, Report of a WHO Expert Committee. 1995: World Health Organization. 
21. FAO, Tropical Livestock Units (TLU). [cited 2019 January 26]; Available: htttp://www.fao.org/ag/againfo/programmes/en/lead/toolbox/Mixed1/TLU.htm\#Exchange.

22. De Onis, M., et al., WHO global database on child growth and malnutrition. Geneva: WHO, 2003.

23. Central Statistical Agency [Ethiopia] and ICF International, Ethiopia Demographic and Health Survey 2016. 2016: Addis Ababa, Ethiopia and Calverton, Maryland, USA.

24. Mengistu, K., K. Alemu, and B. Destaw, Prevalence of malnutrition and associated factors among children aged 6-59 months at Hidabu Abote District, North Shewa, Oromia Regional State. J nutr disorders ther, 2013. 1: p. 1-15.

25. Fentaw, R., A. Bogale, and D. Abebaw, Prevalence of child malnutrition in agro-pastoral households in Afar Regional State of Ethiopia. The Korean Nutrition Society and the Korean Society of Community Nutritio. Samera, Ethiopia. Nutrition Research and Practice (Nutr Res Pract) 2013, 2013. 7(2): p. 9130.

26. Alemayehu, M., et al., Nutritional status and associated factors among under-five children, Tigray, Northern Ethiopia. Int J Nutr Food Sci, 2014. 3(6): p. 579-86.

27. Geberselassie, S.B., et al., Prevalence of stunting and its associated factors among children 6-59 months of age in Libo-Kemekem district, Northwest Ethiopia; A community based cross sectional study. PloS one, 2018. 13(5).

28. Demissie, S. and A. Worku, Magnitude and factors associated with malnutrition in children 6-59 months of age in pastoral community of Dollo Ado district, Somali region, Ethiopia. Science Journal of Public Health, 2013. 1(4): p. 175-178.

29. Demissie, S. and A. Worku, Magnitude and factors associated with malnutrition in children 6-59 months of age in pastoral community of Dollo Ado district, Somali region, Ethiopia. Sci J Public Health, 2013. 1(4): p. 175-83.

30. Zewdu, S., Magnitude and factors associated with malnutrition of children under five years of age in Rural Kebeles of Haramaya, Ethiopia. Harar Bulletin of Health Sciences, 2012. 4: p. 221-232.

31. Tadesse, S.E. and T.C. Mekonnen, Prevalence and Associated Factors of Stunting Among Children Aged 6-59 Months in Delanta District; North East Ethiopia. Nutrition and Dietary Supplements, 2020. 12: p. 41.

32. Organization, W.H., Infant and young child feeding: model chapter for textbooks for medical students and allied health professionals. 2009.

33. Alemayehu, M., et al., Undernutrition status and associated factors in under-5 children, in Tigray, Northern Ethiopia. Nutrition, 2015. 31(7-8): p. 964-970.

34. Takele, K., T. Zewotir, and D. Ndanguza, Understanding correlates of child stunting in Ethiopia using generalized linear mixed models. BMC public health, 2019. 19(1): p. 626.

35. Kandala, N.B., et al., Semiparametric analysis of the socio-demographic and spatial determinants of undernutrition in two african countries. 2001. 
36. Takele, K. and A. Taye, BAYESIAN MODELLING OF GROWTH RETARDATION AMONG CHILDREN UNDER-FIVE YEARS OLD. Far East Journal of Theoretical Statistics, 2014. 48(2): p. 87.

37. Macro, 0., Central Statistical Agency: Ethiopia demographic and health survey 2005. ORC Macro, Calverton, Maryland, USA, 2006.

38. Muldiasman, M., et al., Can early initiation to breastfeeding prevent stunting in 6-59 months old children? Journal of Health Research, 2018.

39. Black, R.E., et al., Maternal and child undernutrition: global and regional exposures and health consequences. The lancet, 2008. 371(9608): p. 243-260.

40. Gebre, A., et al., Prevalence of malnutrition and associated factors among under-five children in pastoral communities of afar regional state, Northeast Ethiopia: A community-based cross-sectional study. Journal of nutrition and metabolism, 2019. 2019.

41. Chirande, L., et al., Determinants of stunting and severe stunting among under-fives in Tanzania: evidence from the 2010 cross-sectional household survey. BMC pediatrics, 2015. 15(1): p. 165.

42. Giashuddin, M., et al., Exclusive breastfeeding and nutritional status in Bangladesh. The Indian Journal of Pediatrics, 2003. 70(6): p. 471-475.

43. Fikadu, T., S. Assegid, and L. Dube, Factors associated with stunting among children of age 24 to 59 months in Meskan district, Gurage Zone, South Ethiopia: a case-control study. Bmc public health, 2014. 14(1): p. 800.

44. Khamis, A.G., et al., The influence of dietary diversity on the nutritional status of children between 6 and 23 months of age in Tanzania. BMC pediatrics, 2019. 19(1): p. 518.

45. Motbainor, A., A. Worku, and A. Kumie, Stunting is associated with food diversity while wasting with food insecurity among underfive children in East and West Gojjam Zones of Amhara Region, Ethiopia. PLoS one, 2015. 10(8).

46. Arimond, M. and M.T. Ruel, Dietary diversity is associated with child nutritional status: evidence from 11 demographic and health surveys. The Journal of nutrition, 2004. 134(10): p. 2579-2585.

\section{Tables}

Table 1. Demographic and socio-economic characteristics of households in Dubti district, Afar region, northeast Ethiopia, January $2018(\mathrm{~N}=554)$ 


\begin{tabular}{|c|c|c|c|}
\hline Variables & Category & Frequency & Percent \\
\hline \multirow[t]{3}{*}{ Head of Household } & Father of the child & 510 & 92.1 \\
\hline & Mother of the child & 25 & 4.5 \\
\hline & Others* & 19 & 3.4 \\
\hline \multirow[t]{4}{*}{ Ethnicity } & Afar & 438 & 79.1 \\
\hline & Amhara & 86 & 15.5 \\
\hline & Oromo & 15 & 2.7 \\
\hline & Others\# & 15 & 2.7 \\
\hline \multirow[t]{3}{*}{ Religion } & Muslim & 513 & 92.6 \\
\hline & Orthodox & 24 & 4.3 \\
\hline & Protestant & 17 & 3.1 \\
\hline \multirow[t]{3}{*}{ Marital status } & Married & 507 & 91.5 \\
\hline & Unmarried & 13 & 2.3 \\
\hline & Divorced/Widowed/separated & 34 & 6.1 \\
\hline \multirow[t]{3}{*}{ Total number of $<5$ children } & 1 & 266 & 48.0 \\
\hline & 2 & 257 & 46.4 \\
\hline & 3 & 31 & 5.6 \\
\hline \multirow[t]{5}{*}{ Educational status of mother } & Can't read and write & 321 & 57.9 \\
\hline & Informal education & 21 & 3.8 \\
\hline & Primary education & 129 & 23.3 \\
\hline & Secondary education & 56 & 10.1 \\
\hline & Higher education & 27 & 4.9 \\
\hline \multirow[t]{4}{*}{ Occupational status of mother } & Housewife & 347 & 62.6 \\
\hline & Agro-pastoralist & 150 & 27.1 \\
\hline & Merchant & 34 & 6.1 \\
\hline & Others§ & 23 & 4.2 \\
\hline \multirow[t]{3}{*}{ Occupational status of father } & Agro-pastoralist & 452 & 81.6 \\
\hline & Merchant & 68 & 12.3 \\
\hline & Government/self-employee & 34 & 6.1 \\
\hline
\end{tabular}




\begin{tabular}{|llll|} 
Family size & $<5$ & 251 & 45.3 \\
\hline Wealth status & $\geq 5$ & 303 & 54.7 \\
& Poor & 412 & 74.4 \\
& Medium & 81 & 14.6 \\
\hline PSNP user & Rich & 61 & 11.0 \\
& No & 514 & 92.8 \\
& Yes & 40 & 7.2 \\
\hline
\end{tabular}

Note: Others: *Care givers of the targeted child; \# Tigre, Wolayita, Somali; § Private organization employee, government employee, student, NGO employee. PSNP = Productive safety net programme

Table 2. Child characteristics and child carrying practice in Dubti district, Afar region, northeast Ethiopia, January $2018(\mathrm{~N}=554)$ 


\begin{tabular}{|c|c|c|c|}
\hline Variables & Category & Frequency & Percent \\
\hline \multirow[t]{2}{*}{ Sex of the child } & Female & 242 & 43.7 \\
\hline & Male & 312 & 56.3 \\
\hline \multirow[t]{4}{*}{ Age of the child in months } & $6-11$ & 111 & 20.0 \\
\hline & $12-23$ & 122 & 22.0 \\
\hline & $24-35$ & 177 & 31.9 \\
\hline & $36-59$ & 144 & 26.0 \\
\hline \multirow[t]{4}{*}{ Birth order } & First & 122 & 22.0 \\
\hline & Second & 198 & 35.7 \\
\hline & Third & 110 & 19.9 \\
\hline & Fourth and above & 124 & 22.4 \\
\hline \multirow[t]{2}{*}{ Type of birth } & Single & 549 & 99.1 \\
\hline & Twin & 5 & 0.9 \\
\hline \multirow[t]{2}{*}{ Place of delivery } & Home & 291 & 52.5 \\
\hline & Health institution & 263 & 47.5 \\
\hline \multirow[t]{2}{*}{ Time of initiation of breast feeding } & Within 1 hour & 439 & 79.2 \\
\hline & After 1 hour & 115 & 20.8 \\
\hline \multirow[t]{2}{*}{ Exclusive breast feeding } & $<6$ months & 108 & 19.5 \\
\hline & $\geq 6$ months & 446 & 80.5 \\
\hline \multirow[t]{2}{*}{ Frequency of feeding } & $<3$ times & 251 & 45.3 \\
\hline & $\geq 3$ times & 303 & 54.7 \\
\hline \multirow[t]{2}{*}{ Minimum dietary diversity score } & $<4$ & 461 & 83.2 \\
\hline & $\geq 4$ & 93 & 16.8 \\
\hline \multirow[t]{3}{*}{ Immunization status } & Not vaccinated & 67 & 12.1 \\
\hline & Partially vaccinated & 173 & 31.2 \\
\hline & Fully vaccinated & 314 & 56.7 \\
\hline \multirow[t]{3}{*}{ Morbidity } & No disease & 190 & 34.3 \\
\hline & One disease & 253 & 45.7 \\
\hline & Two and more diseases & 111 & 20.0 \\
\hline
\end{tabular}


Table 3. Maternal characteristics in Dubti district, Afar region, northeast Ethiopia, January $2018(\mathrm{~N}=554)$

\begin{tabular}{|llll|}
\hline Variables & Category & Frequency & Percent \\
\hline Age of mother & $\leq 25$ & 201 & 36.3 \\
\hline Number of ANC visit & $26-35$ & 279 & 50.4 \\
\hline & $\geq 36$ & 74 & 13.3 \\
\hline PNC follow up & 0 & 36 & 6.5 \\
\hline Extra meal given to mother during pregnancy or lactation & No & 240 & 43.3 \\
\hline & Yes & 278 & 50.2 \\
\hline Decision making on use of money & No & 138 & 24.9 \\
\hline & Mainly spouse & 81 & 75.1 \\
\hline & Mainly husband & 395 & 71.3 \\
\hline
\end{tabular}

Table 4. Environmental health characteristics of households in Dubti district, Afar region, northeast Ethiopia, January $2018(\mathrm{~N}=554)$ 


\begin{tabular}{|llll|}
\hline Variables & Category & Frequency & Percent \\
\hline Source of drinking water & River & 150 & 27.1 \\
\hline & Spring & 59 & 10.6 \\
\hline Time to obtain drinking water (round trip) & Public tap & 345 & 62.3 \\
\hline & 15 minutes & 267 & 48.2 \\
\hline Latrine utilization & $>30$ minutes & 160 & 28.9 \\
\hline & No & 127 & 22.9 \\
\hline Materials used for hand washing & Yes & 328 & 40.8 \\
\hline & Water only & 214 & 39.2 \\
\hline & Using soap sometimes & 227 & 41.0 \\
\hline & Using soap always & 113 & 20.4 \\
\hline
\end{tabular}

Table 5. Factors affecting stunting among children aged between 6 and 59 months in Dubti district, Afar region, northeast Ethiopia, January $2018(\mathrm{~N}=554)$ 


\section{Variables}

\section{Economic status}

Poor

Medium

Rich

\section{Sex of the child}

Female
Male
Age of the child

\section{6-11}

$12-23$

24-35

36-59

Initiation of breast feeding

After 1 hour

Within 1 hour

\section{Exclusive breast feeding}

$\leq 6$ months

$>6$ months

\section{Frequency of feeding per day}

$<3$ times

3 times

$>3$ times

Minimum dietary diversity score

$\leq 4$

$>4$

Immunization status of the child

Not vaccinated

Stunting COR with $95 \% \mathrm{Cl} \quad$ AOR with $95 \% \mathrm{Cl}$

Yes No

$\begin{array}{llll}186 & 226 & 4.2(2.07,8.49)^{\star \star \star} & 5.50(2.52, \\ & & & 12.04)^{\star \star \star} \\ 23 & 58 & 2.02(0.88,4.65) & 2.38(0.96,5.93) \\ 10 & 51 & 1 & 1\end{array}$

$87 \quad 155 \quad 0.76(0.54,1.08) \quad 0.67(0.45,1.01)$

$\begin{array}{lll}132 & 180 & 1\end{array}$

1

$\begin{array}{llll}16 & 95 & 1 & 1\end{array}$

$4280 \quad 3.12(1.63,5.96)^{\star \star} \quad 2.55(1.27,5.09)^{\star \star}$

$73104 \quad 4.17(2.27,7.66)^{\star \star \star} \quad 3.02(1.58,5.78)^{\star \star}$

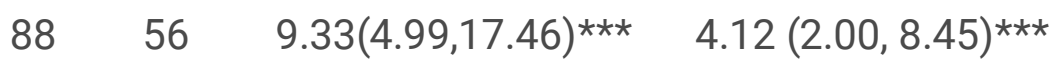

$66492.52(1.66,3.83)^{\star \star \star} \quad 1.99(1.22,3.23)^{\star \star}$

$\begin{array}{lll}153 & 286 & 1\end{array}$

1

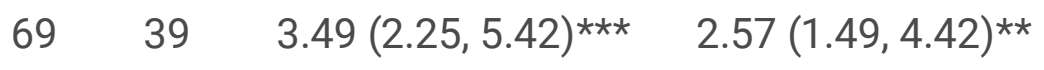

$\begin{array}{llll}150 & 296 & 1 & 1\end{array}$

\begin{tabular}{llll}
104 & 147 & $1.54(0.95,2.50)$ & $1.45(0.83,2.54)$ \\
\hline 82 & 116 & $1.54(0.94,2.54)$ & $1.75(0.98,3.12)$ \\
33 & 72 & 1 & 1
\end{tabular}

$2022593.49(1.99,6.09)$ *** $1.93(1.03,3.62)^{*}$

$\begin{array}{llll}17 & 76 & 1 & 1\end{array}$

$3532 \quad 1.89(1.11,3.22)$ * $\quad 0.73(0.37,1.43)$ 


\begin{tabular}{|lllll|}
\hline Partially vaccinated & 69 & 104 & $1.15(0.78,1.68)$ & $0.64(0.34,1.21)$ \\
\hline Fully vaccinated & 115 & 199 & 1 & 1 \\
\hline $\begin{array}{l}\text { Time to obtain drinking water (round } \\
\text { trip) }\end{array}$ & & & & 1 \\
\hline$<15$ minutes & 88 & 179 & 1 & $1.56(0.96,2.55)$ \\
\hline $15-30$ minutes & 67 & 93 & $1.46(0.98,2.19)$ & $1.52(0.79,2.91)$ \\
\hline$>30$ minutes & 64 & 63 & $2.07(1.34,3.18)^{\star \star}$ & \\
\hline Materials used for hand washing & & & & $1.29(0.74,2.25)$ \\
\hline Only water & 103 & 111 & $1.69(1.06,2.71)^{\star}$ & $1.03(0.59,1.78)$ \\
\hline Using soap sometimes & 76 & 151 & $0.92(0.57,1.48)$ & 1 \\
\hline Always using soap and water & 40 & 73 & 1 & $1.68(1.21,2.94) \star$ \\
\hline Source of drinking water & & & & $1.12(0.41,1.96)$ \\
\hline River & 82 & 68 & $2.41(1.63,3.57) \star \star \star$ & 1 \\
\hline Spring & 22 & 37 & $1.19(0.67,2.11)$ & 1 \\
\hline Public tap & 115 & 230 & 1 & 1 \\
\hline
\end{tabular}

\section{Figures}




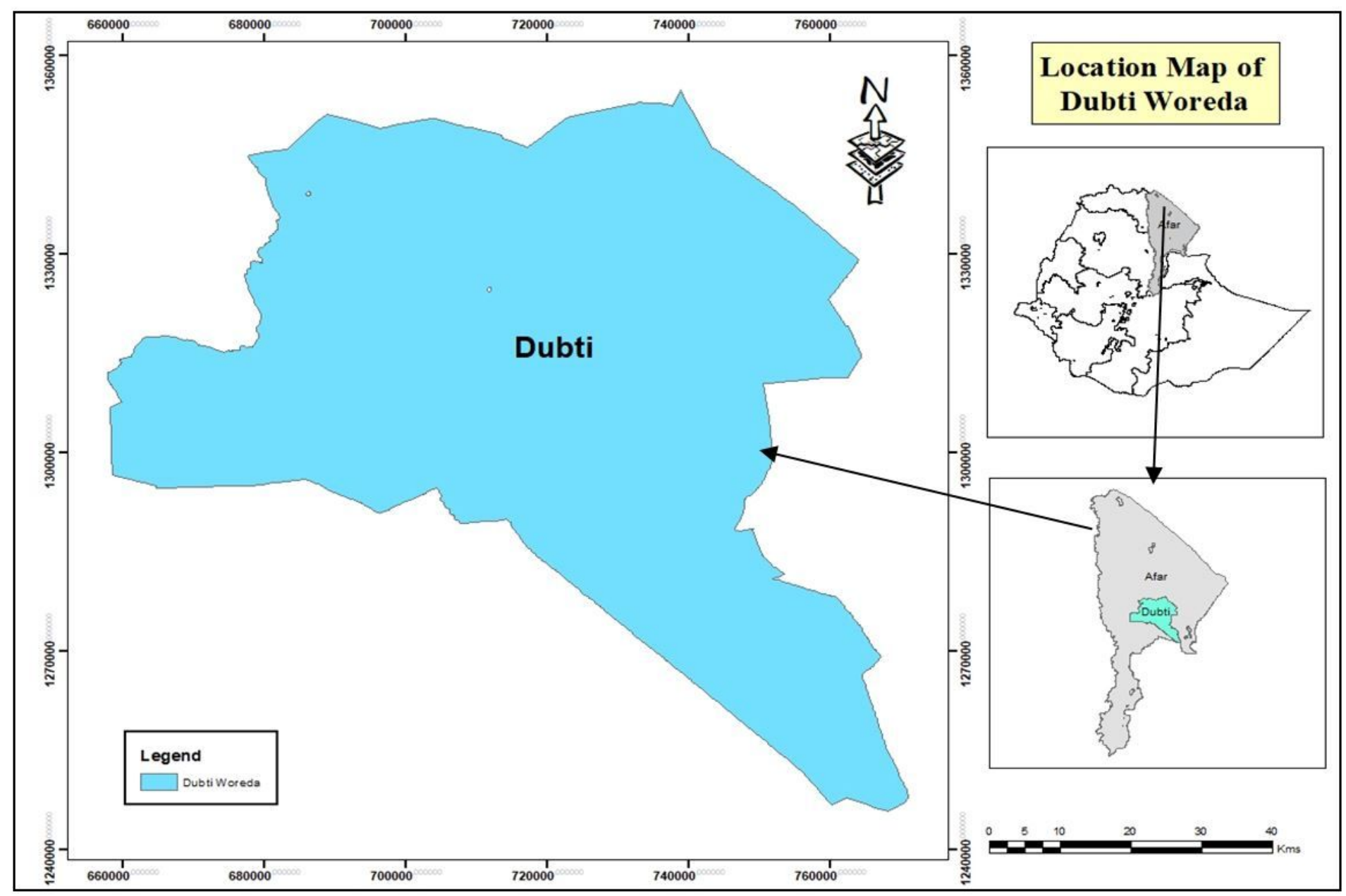

Figure 1

Map of the study area. 